\title{
Employing Cathodoluminescence for Nanothermometry and Thermal Transport Measurements in Semiconductor Nanowires
}

\author{
Kelly W. Mauser,* Magdalena Solà-Garcia, Matthias Liebtrau, Benjamin Damilano, Pierre-Marie Coulon, \\ Stéphane Vézian, Philip A. Shields, Sophie Meuret, and Albert Polman*
}

Cite This: ACS Nano 2021, 15, 11385-11395

Read Online

ABSTRACT: Thermal properties have an outsized impact on efficiency and sensitivity of devices with nanoscale structures, such as in integrated electronic circuits. A number of thermal conductivity measurements for semiconductor nanostructures exist, but are hindered by the diffraction limit of light, the need for transducer layers, the slow scan rate of probes, ultrathin sample requirements, or extensive fabrication. Here, we overcome these limitations by extracting nanoscale temperature maps from measurements of bandgap cathodoluminescence in $\mathrm{GaN}$ nanowires of $<300 \mathrm{~nm}$ diameter with spatial resolution limited by the electron cascade. We use this thermometry method in three ways to determine the thermal conductivities of the nanowires in the range

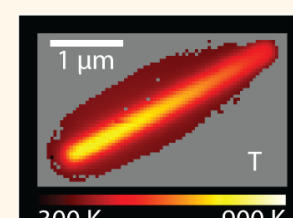

$300 \mathrm{~K}$ $900 \mathrm{~K}$ electron beam of $19-68 \mathrm{~W} / \mathrm{m} \cdot \mathrm{K}$, well below that of bulk GaN. The electron beam acts simultaneously as a temperature probe and as a controlled delta-function-like heat source to measure thermal conductivities using steady-state methods, and we introduce a frequency-domain method using pulsed electron beam excitation. The different thermal conductivity measurements we explore agree within error in uniformly doped wires. We show feasible methods for rapid, in situ, high-resolution thermal property measurements of integrated circuits and semiconductor nanodevices and enable electron-beam-based nanoscale phonon transport studies.

KEYWORDS: cathodoluminescence thermometry, nanothermometry, thermal transport, thermal conductivity, gallium nitride nanowire, semiconductor nanowire, cathodoluminescence

$\mathrm{H}$ ighly concentrated energy densities and fewer thermal conduction pathways through which waste heat dissipates can lead to substantially elevated temperatures and thereby reduced performance in nanodevices. Temperature control is crucial in many systems: nanowire single-photon detectors must be cryogenically cooled to enter the superconducting regime and eliminate thermal noise; ${ }^{1}$ nanowire lasers see a shift in lasing threshold and wavelength with temperature rises; ${ }^{2}$ thermoelectric nanostructures rely on low thermal conductivity to generate large temperature gradients to increase efficiency of power generation or detection; ${ }^{3}$ and a microchip can have significantly varying gain and noise characteristics across its range of operating temperatures. Additionally, as integrated circuits shrink in size, the on-chip power density has increased by an order of magnitude over a decade, creating challenges in how to handle heat dissipation in nanoscale transistors. ${ }^{4}$ Therefore, careful design of thermal management in nanostructure devices is critical to provide stable output and performance, as these technologies increasingly move to smaller scales. Past research has studied engineering thermal conductivity by measuring or tailoring phonon mean free path spectra. ${ }^{5-13}$ However, measuring both temperature and thermal conductivity of nanostructures is notoriously difficult.

A number of noninvasive methods have been devised to measure temperature and thermal conductivity on the nanoscale. $^{14-17}$ The highest spatial resolution thermometry methods include near-field scanning optical microscopy, ${ }^{18,19}$ scanning thermal microscopy, ${ }^{20,21}$ and transmission electron microscopy. ${ }^{22-24}$ These methods can have a spatial resolution

Received: January 29, 2021

Accepted: June 16, 2021

Published: June 22, 2021 


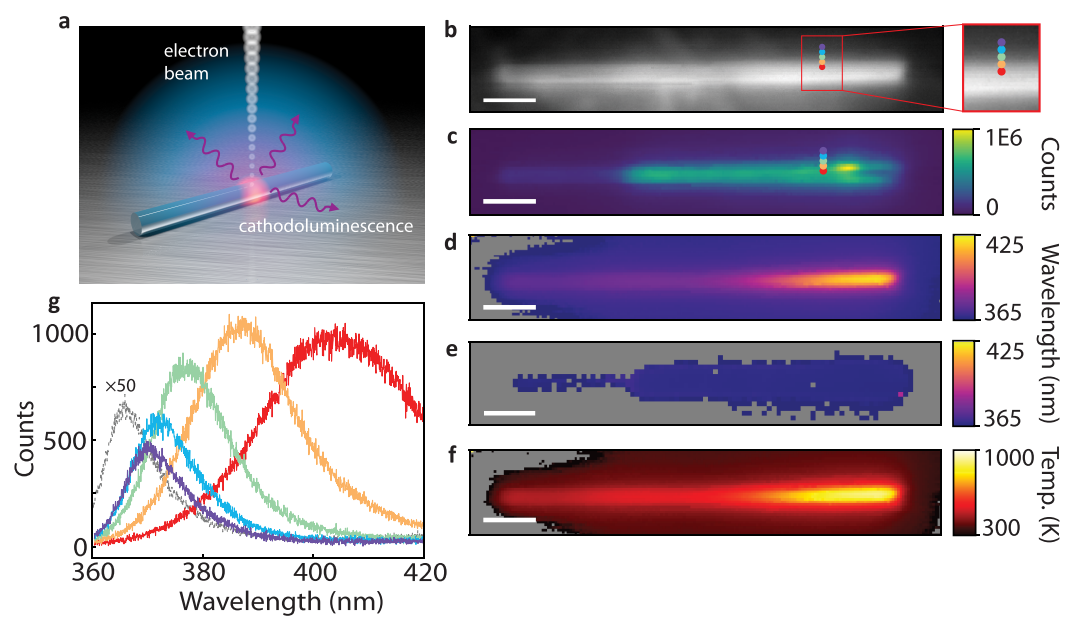

Figure 1. Nanoscale thermometry CL measurement technique and monitored signals. (a) Schematic of cathodoluminescence (CL) measurements on a semiconductor nanowire. An electron beam heats/excites the semiconductor nanowire, and incoherent CL is collected by the high-numerical-aperture parabolic mirror and directed into a spectrometer. (b) SEM image $(210 \mathrm{nA} 5 \mathrm{keV}$ electron beam) of a GaN nanowire taken simultaneously with CL data. (Inset) Zoomed-in region of a GaN wire. (c) CL counts integrated between the wavelengths of 360-420 nm. (d, e) Peak CL wavelength extracted by fitting the spectra corresponding to each pixel with a Lorentzian for experiments performed with an electron beam current of $210 \mathrm{nA}(\mathrm{d})$ or $1.6 \mathrm{nA}(\mathrm{e})$. Gray regions were pixels with peaks of less than 10 counts. (f) Temperature map measured when the electron beam $(210 \mathrm{nA})$ is focused at each pixel, obtained by fitting the data in (d) to eq 1 . (g) CL spectra. Each spectrum is obtained at the position of the corresponding color dot in both (b) and (c). The amount of spatial overlap of the electron beam and the nanowire dictates the energy absorbed in the nanowire from the electron beam, resulting when the beam is centered on the nanowire, in a maximum temperature rise and corresponding red-shift of the CL emission according to eq 1 . The gray curve corresponds to CL taken from a $1.6 \mathrm{nA}$ electron beam at the location of the red dot (using a $50 \times$ longer exposure time), and the other curves were taken with a $210 \mathrm{nA}$ electron beam. Scale bars are $500 \mathrm{~nm}$.

well below $100 \mathrm{~nm}$ but have generally slow data collection or cumbersome probes or require very thin samples. Common thermal conductivity measurement methods include the $3 \omega$ method, ${ }^{25,26}$ the suspended microchip method, ${ }^{27-29}$ and timedomain thermoreflectance ${ }^{8,9,13,30-33}$ and its variants. ${ }^{33}$ These methods lack the spatial resolution of the high-resolution thermometry methods listed above and require invasive or extensive fabrication or a transducer layer, all of which hinders the ability to measure smaller domains or thermal boundary effects. $^{31,33}$

Here we demonstrate a rapid, high-resolution solution for measuring temperature and the thermal conductivity of nanostructures using cathodoluminescence (CL) spectroscopy. $\mathrm{CL}$ is radiation emitted when a high-energy electron beam (e.g., in a scanning electron microscope (SEM) or transmission electron microscope) interacts with a material. In semiconductors, CL derives primarily from bandgap emission generated when high-energy electrons lose energy to bulk plasmons through inelastic collisions, which then excite hot electron hole pairs, which can thermalize and/or generate electron hole pairs that subsequently recombine by the emission of CL. ${ }^{34-36} \mathrm{CL}$ spectroscopy has been used in mineralogy, ${ }^{37}$ semiconductor characterization, ${ }^{38,39}$ and the study of plasmonic and photonic modes in metallic or dielectric nanostructures with nanometer resolution. ${ }^{40,41}$ As we will show, at low beam currents, CL thermometry provides high-resolution noninvasive temperature measurements. At high beam currents, the beam acts like a nearly delta function heat source while simultaneously probing the temperature. CL has seldom been used for thermometry, ${ }^{42,43}$ and it has not, to our knowledge, been used for nanoscale thermal imaging or to study thermal conductivity. In our method of CL thermometry, the spatial resolution is limited primarily by the electron beam cascade size in the material, which can be in the range of about 1-200 $\mathrm{nm}$ depending on the electron energy, and also by the electron probe size and the minority carrier diffusion length. ${ }^{44}$ We apply this technique to study the thermal conductivity of $\mathrm{GaN}$ nanowires, which are of increased interest due to their promise for optoelectronic applications ${ }^{45,46}$ including nanowire lasers. ${ }^{47-49}$

First, with the spatial resolution of an SEM cascade, we use the thermal bandgap shift in semiconductors to map out the temperature profile from an electron-beam-induced heat source in a nondestructive manner, measuring electron-beaminduced temperature rises of over $500 \mathrm{~K}$. Next, we use this thermometry technique to extract the thermal conductivity of $\mathrm{GaN}$ nanowires with three different methods: two methods using a DC electron beam current, and one method using a technique involving an ultrafast electron beam blanker to provide an AC heating/thermometry source. The data obtained using the three methods is comparable in finding that the GaN nanowires exhibit a thermal conductivity lower than that of bulk GaN. With higher spatial resolution than state-of-the-art laser-based techniques ${ }^{32}$ combined with fast scan speeds, variable probing/heating depth, and no near-field probe or thin sample requirement, our work enables nanoscale phononic and thermal transport studies in semiconductors, including in situ measurements of silicon-integrated circuits.

\section{RESULTS AND DISCUSSION}

Temperature Measurements. The concept of CL is shown in Figure 1a, and the CL setup used is described further in previous work. ${ }^{50}$ Briefly, a parabolic mirror inside of the SEM chamber with a numerical aperture of $1.46 \pi \mathrm{sr}$ is aligned over the sample so that the focal point of the mirror corresponds to the electron beam focus on the sample, and light is then collected and directed to a spectrometer. An SEM image of one of the GaN nanowires used is shown in Figure 
1b. The 200-300 nm diameter GaN nanowires were made by a top-down approach based on sublimation under vacuum (see Methods) and exhibit lasing properties ${ }^{49}$ under optical pumping. We observe that the nanowires continue lasing while simultaneously undergoing high current electron beam irradiation indicating negligible degradation of the wires during CL measurements (see Figure S1 for more details). The nanowire was broken off the substrate on which it was grown and placed on the frame of a copper TEM grid covered with a $2 \mathrm{~nm}$ thickness of lacey carbon film, which thermally isolates the wire relatively well. Figure 1c shows a CL intensity map of the wire for emission between 360 and $420 \mathrm{~nm}$, and the corresponding SEM image (collected during CL imaging) is shown in Figure $1 \mathrm{~b}$ taken at a beam current of $210 \mathrm{nA}$ and electron energy of $5 \mathrm{keV}$. At each pixel in the CL map, the electron beam is focused at this point, and light is collected and the spectrum analyzed. Figure $1 \mathrm{~g}$ illustrates a red-shift in the peak bandgap emission of the CL spectrum as the electron beam becomes more centered on the wire, which we will explain shortly, and the color of the spectra in Figure $1 \mathrm{~g}$ corresponds to the location of the colored dots on Figure $1 b, c$ (the gray curve is CL from a lower current $1.6 \mathrm{nA}$ beam at the location of the red dot). The small, non-red-shifted CL peak observed for the beam placed next to the wire results from either backscattered or secondary electrons from the substrate, which excite CL from the wire and deposit little power inside, or stray electrons from an enlarged electron beam. The most intense and most red-shifted CL is observed for a high-current electron beam centered on the wire. Using CASINO ${ }^{51}$ Monte Carlo simulations, we determine that approximately $71 \%$ of the energy of the electrons is converted to heat in the wire. The remainder of the electron beam energy is mostly lost to backscattered and secondary electrons (Figure S2), with a negligible amount of energy lost to bandgap emission (Figure S3) and X-rays. Electron beam heating ${ }^{52-55}$ calculations based on CASINO Monte Carlo simulations have previously been verified experimentally. ${ }^{52,53}$ With a $210 \mathrm{nA}$ electron beam, this corresponds to $746 \mu \mathrm{W}$ of power being deposited in the wire in a nearly delta-function-shaped power distribution (see Figure S2).

Our thermometry is carried out by tracking the shift in the peak bandgap emission energy as a function of temperature, due to thermal expansion of the lattice and changes in electron-phonon interactions with temperature. ${ }^{56,57}$ As shown in Figure S4, we calibrate the wavelength shift with temperature in our GaN nanowires by measuring the bandgap shift as a function of temperature between 90 and $300 \mathrm{~K}$ using a 548 pA beam current in a liquid-nitrogen-cooled cryogenic stage on our microscope. At a beam current this low the heating induced by the electron is negligible. While GaN shows a bandgap shift at room temperature and higher, many of our measurements were carried out below room temperature within the range of our calibration curve to ensure our measurements were accurate (we did not have a heating stage available for calibration); in some cases we extrapolate this curve to higher temperatures, following the Varshni phenomelogical formula, 57

$$
E_{\mathrm{g}}(T)=E_{\mathrm{g}}(0)-\frac{\gamma T^{2}}{\beta+T}
$$

where $E_{\mathrm{g}}$ is the bandgap as a function of temperature, $T, E_{\mathrm{g}}(0)$ is the bandgap energy at $0 \mathrm{~K}$ (a fit parameter), and $\gamma$ and $\beta$ are constants. ${ }^{57}$ The bandgap shift could alternatively be fit to an expression from O'Donnell and Chen. ${ }^{56}$ While we focus on CL thermal measurements in $\mathrm{GaN}$ in this paper, a bandgap shift (red or blue) with increasing temperature can be seen in many other semiconductor materials; ${ }^{56,57} \mathrm{CL}$ spectra for intrinsic $\mathrm{GaAs}$ and p-doped $\mathrm{Si}$ wafers at different temperatures are shown in Figure S4 to demonstrate that the CL thermometry and the thermal conductivity measurement techniques presented here are not limited to use with GaN. Photoluminescence bandgap shifts in $\mathrm{GaN}$ nanowires have previously been used to measure temperature in a similar manner, but suffer from the poor resolution of the laser used as a heater/probe. ${ }^{58}$

From our fit (Figure S4), we determined $E_{g}(0)=3.471 \mathrm{eV}$ (in $\mathrm{GaN}$ this corresponds to a donor-bound excitonic transition at low temperature, not the bandgap ${ }^{59}$ ), $\beta=2609$ $\mathrm{K}$, and $\gamma=2.25 \times 10^{-3} \mathrm{eV} / \mathrm{K}$, which is similar to previous studies of $\mathrm{GaN}$ epilayers on sapphire substrates, ${ }^{59}$ with differences likely caused by different growth mechanism and the nanoscale geometries, and the fact that we fit a single Lorentzian to the entire near-band-edge PL spectrum to determine our effective bandgap instead of tracking shifts of individual exciton transitions the PL spectrum is composed of. The root-mean-square error in temperature of our data around the line of best fit is $6.0 \mathrm{~K}$, which was measured in a region of fairly uniform doping. The thermal stage used had a temperature accuracy of $\pm 1 \mathrm{~K}$, and additional error likely comes from small doping variations within the region measured. Larger errors in temperature will arise in regions of dissimilar doping, which can be corrected for and will be discussed later. We fit the data in Figure $1 \mathrm{~d}$ with eq 1 to create a temperature map (Figure 1f) of the GaN nanowire resulting from electron beam heating at each pixel.

In order to reduce uncertainty in the thermal contact area between the wires and the substrate, nanowires were scattered over a copper TEM grid (Ted Pella G2000HA) with $6.5 \mu \mathrm{m}$ diameter holes. Wires that straddled holes were heat sunk to the copper via electron beam assisted Pt deposition to fix the temperature at the ends of the wires during heating and reduce interfacial thermal resistance between the wires and the $\mathrm{Cu}$ TEM grid. ${ }^{28}$ An SEM image of a wire in this configuration is shown in Figure 2a. Following previous work, ${ }^{27-29,52-55,58,60,61}$ we treat the nanowires as 1D systems and ignore thermal radiation and losses from CL (see Figure S3) in our analytical calculations. Finite element ${ }^{62}$ simulations support this approximation. Figure $2 \mathrm{~b}-\mathrm{e}$ show temperature maps of the wire in Figure 2a for different electron beam currents, in which the electron beam itself is used both as a heat source and as a thermometer. In these maps, the sample stage temperature was maintained at $161 \mathrm{~K}$, and the peak CL wavelength was extracted for each pixel on the map and converted to temperature according to our calibration curve (eq 1). Note that each pixel is measured when the electron beam is focused on that particular location. We use a $5 \mathrm{keV}$ electron beam, as Monte Carlo simulations indicate that at this energy most of the electron energy will be deposited within the wire (Figure S2). A higher energy beam would give better spatial resolution, but most of the electrons would pass through the wire without interacting, decreasing heat deposition.

Several trends can be observed from the data in Figure 2. First, in all images the largest temperature rise is observed toward the center of the wire, as expected theoretically for a 1D system with fixed temperature at both boundaries and an 


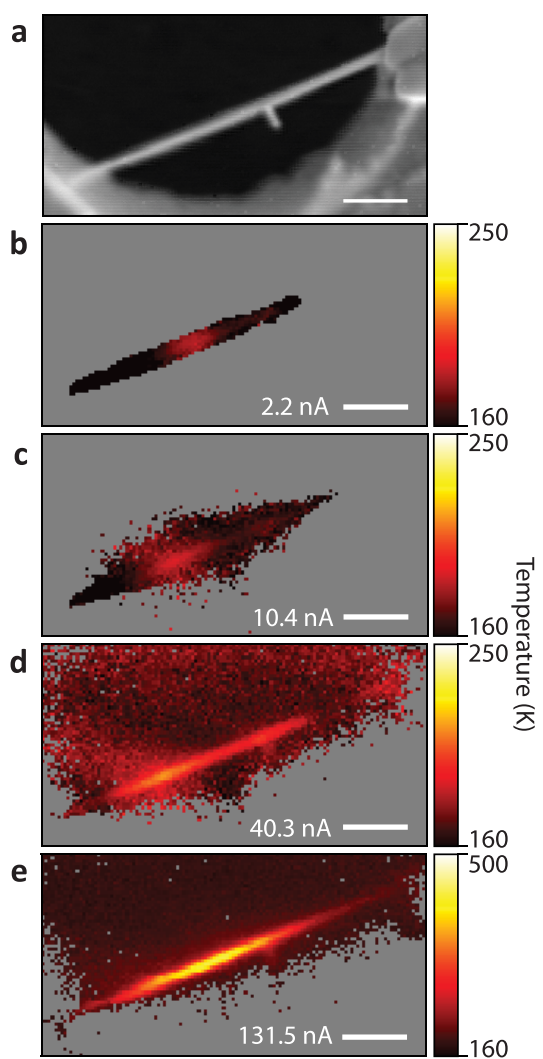

Figure 2. Nanoscale temperature measurements at variable electron beam currents. (a) SEM of suspended GaN nanowire with Pt heat sinks on either end. $(b-e)$ Temperature measurements of a GaN nanowire at the specified electron currents. Gray regions indicate pixels that did not exhibit a peak in the CL spectrum above 100 counts and $1 \mathrm{~nm}$ in width or which could not be fit. Scale bars are $1 \mu \mathrm{m}$. The base temperature in all measurements is $161 \mathrm{~K}$.

internal heat source. We can also see the high spatial resolution of the CL thermometry technique. Using higher beam currents we can generate temperature increases of over $200 \mathrm{~K}$, showing the power of this technique to create temperature profiles from which the thermal conductivity can be derived, as we will show below. The CL from this wire was comparatively weak (possibly due to either the doping level or increased surface recombination velocity from carbon deposition), so a measurement with low enough current to create a nonheated background measurement was not possible. In order to produce the large currents in Figure $2 \mathrm{c}-\mathrm{e}$, a large $1 \mathrm{~mm}$ aperture in the SEM column was used, which blocked fewer astigmatic electrons and created a misshapen electron beam. This decreased resolution meant that when the majority of the electron beam was focused on a pixel in the hole of the TEM grid, some stray electrons were still striking the wire. Different electron beam focusing settings (spots) were used to generate different currents, which likely caused the hot spot in the center of the wire to shift slightly. Figure $2 b$ used the same electron beam focusing settings as in Figure 2e, but used a 100 $\mu \mathrm{m}$ aperture.

DC Thermal Conductivity Measurements. We demonstrate three different methods to derive the thermal conductivity from the $\mathrm{CL}$ profiles, the first two being $\mathrm{DC}$ measurements, with analysis similar to Raman thermography or photoluminescence mapping found in other work. ${ }^{58,60,61}$ In the DC measurement techniques, the wire is suspended over a hole in the TEM grid as shown in Figure $2 \mathrm{a}$ and Figure $3 \mathrm{~g}$ and heated by a continuous electron beam, and the steady-state temperature is extracted at every point along the wire as shown in Figure 3a,b. We fit the temperature profile using theoretical models for $1 \mathrm{D}$ wires with the temperature fixed by heat sinking with SEM-deposited $\mathrm{Pt}$ at both ends (the bridge method, Figure $3 \mathrm{c}$ ) or at one end (the slope method, Figure $3 \mathrm{e}$ ). In the bridge method, thermal contact resistance between the $\mathrm{GaN}$ and $\mathrm{Cu}$ TEM grid must be negligible, ${ }^{61}$ and in the slope method this thermal contact resistance is not important. ${ }^{60}$

In the DC bridge method, both ends of the nanowire are heat sunk with SEM-deposited $\mathrm{Pt}$ and suspended over a bare copper TEM grid hole (see inset of Figure 3a,g). We form an equivalent resistance model for the wire (described in more detail in a Supplementary Note), shown in Figure 3d, similar to previous work. ${ }^{61} \mathrm{We}$ assume there are two different thermal conductivities in the system: the thermal conductivity of $\mathrm{GaN}$ in the center of the wire, $\kappa_{\mathrm{GaN}}$, and an effective thermal conductivity for a mixture of $\mathrm{GaN}$ and $\mathrm{Pt}$ closer to the Pt heat sinks, $\kappa_{0}$, attributed to the enlarged $\mathrm{GaN}$ nanowire radius due to excess $\mathrm{Pt}$ on the surface (see Figure S5, Figure 3c). $L_{1}$ and $L_{2}$ demarcate the boundaries between the regions of different thermal conductivities and were treated as fit parameters. The system is represented in Figure $3 \mathrm{~d}$ by a thermal circuit model. Here, the thermal resistance is given by $R=l \mathrm{~A} / \kappa$, with $l$ being the relevant length of the particular segment and $\kappa$ the thermal conductivity of that segment. $l$ can change depending on the position of the heat source (see Figure 3c, Supplementary Note), so the equations for the peak temperature rise, $\Delta T(x)$, as a function of $x$, the position of the electron beam heat source/thermometer, are

$$
\begin{aligned}
& \Delta T(x)=\frac{\dot{Q}}{A}\left(\frac{\kappa_{0}}{x}+\frac{\kappa_{0} \kappa_{\mathrm{GaN}}}{\kappa_{\mathrm{GaN}}\left(L_{1}-x+L-L_{2}\right)+\kappa_{0}\left(L_{2}-L_{1}\right)}\right)^{-1}, 0 \leq x \leq L_{1} \\
& \Delta T(x)=\frac{\dot{Q}}{A \kappa_{0} \kappa_{\mathrm{GaN}}}\left(\frac{1}{\kappa_{\mathrm{GaN}} L_{1}+\kappa_{0}\left(x-L_{1}\right)}+\frac{1}{\kappa_{0}\left(L_{2}-L_{1}\right)+\kappa_{\mathrm{GaN}}\left(L-L_{2}\right)}\right)^{-1}, L_{1} \leq x \leq L_{2} \\
& \Delta T(x)=\frac{\dot{Q}}{A}\left(\frac{\kappa_{0} \kappa_{\mathrm{GaN}}}{\kappa_{\mathrm{GaN}}\left(L_{1}+x-L_{2}\right)+\kappa_{0}\left(L_{2}-L_{1}\right)}+\frac{\kappa_{0}}{L-x}\right)^{-1}, L_{2} \leq x \leq L
\end{aligned}
$$

where $\dot{Q}$ is the heat flux from the electron beam, $L$ is the total wire length, $A$ is the cross-sectional area of the wire as measured via SEM images, and $\Delta T(x)=T(x)-T_{0}$, where $T_{0}$ is the fixed temperature at $x=0$ and $x=L$. Figure $3 c$ shows this geometry in more detail. We fit temperature data obtained in Figure $2 \mathrm{e}$ with eq 2 in order to extract $\kappa_{\mathrm{GaN}}, \kappa_{0}, L_{1}$, and $L_{2}$. 

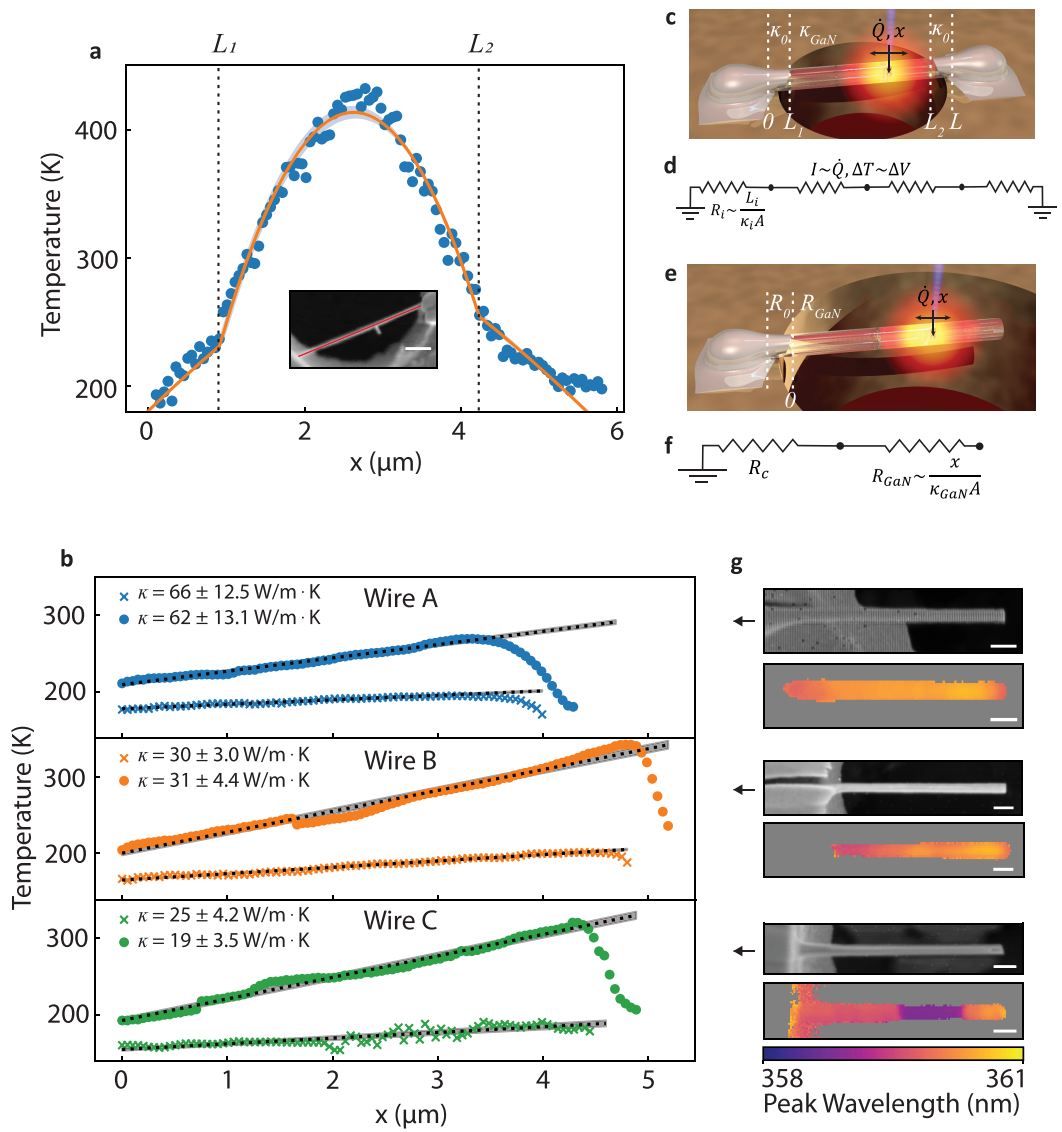

Figure 3. Probing nanowire thermal conductivity with a DC electron beam. (a) Measured temperature as a function of position along the cut through the GaN wire from Figure 2a (shown in the inset). Orange line is best fit to data using eq 2 (DC bridge method), and blue shading is 1 standard deviation of the fit error. We find a thermal conductivity of the GaN nanowire of $22 \pm 4.7 \mathrm{~W} / \mathrm{m} \cdot \mathrm{K}$ and of the $\mathrm{Pt} / \mathrm{GaN}$ portion 91 $\pm 18.9 \mathrm{~W} / \mathrm{m} \cdot \mathrm{K}$. Base temperature for these measurements is $161 \mathrm{~K}$. Wire radius is $118 \mathrm{~nm}$. (b) Demonstration of DC slope method for determining thermal conductivity of two different nanowires with fixed temperature at one end. " $X$ " data points are from $100 \mu \mathrm{m}$ apertured electron beams with $\mathrm{nm}$ spot sizes. The " $O$ " data points are from data collected with $1 \mathrm{~mm}$ apertured electron beams, which result in a less well-defined spot size. The corresponding thermal conductivities are shown in the legends. Radius of the nanowires is $130 \pm 11.8,123 \pm 5.8$, and $142 \pm 11.4 \mathrm{~nm}$ for wires A, B, and C, respectively. (c) Schematic of temperature profile in the wire corresponding to the DC bridge method and values in eq 2. (d) Thermal circuit model for the DC bridge method, shown here for the case of $L_{1} \leq x \leq L_{2}$, where $x$ is the location of the electron beam (see Supplementary Note for more details). (e) Schematic of the temperature profile in the wire corresponding to the DC slope method and values in eq 3. (f) Thermal circuit model for the DC slope method. (g) SEM and peak wavelength map for each wire in the plot on the left. The peak wavelength is measured with an electron beam current of $1 \mathrm{nA}$ to extract the doping variation without significantly heating the nanowire. The wavelength shift due to doping was subtracted from wavelength shifts due to heating to produce the curves in (b); see Supplementary Figure S6. The apparent crack in the Pt in the SEM images is due to Pt being deposited at an angle to ensure good thermal contact between the wire and $\mathrm{Cu}$ below by filling gaps on one side of the wire. Scale bars are $500 \mathrm{~nm}$.

This fit is shown with the data in Figure $3 \mathrm{a}$. We find the thermal conductivity of the $\mathrm{GaN}$ region to be $\kappa_{\mathrm{GaN}}=22 \pm 4.7$ $\mathrm{W} / \mathrm{m} \cdot \mathrm{K}$ and the thermal conductivity of the edge region to be $\kappa_{0}=91 \pm 18.9 \mathrm{~W} / \mathrm{m} \cdot \mathrm{K}$.

Several factors affect the accuracy of the determination of the parameters in the DC bridge method. First of all, our calibration curve only extends up to room temperature, while we extrapolate above room temperature in this analysis, creating some uncertainty. In future work this can be avoided by performing a more extended calibration. Second, a small variation in doping within each nanowire causes a $\sim 1 \mathrm{~nm}$ variation in CL peak energy in different places along the wire ( $C L$ variation due to doping has also been observed previously in GaAs nanowires ${ }^{39}$ ), which also affects the temperature calibration. This could be corrected for by using, as a reference, low-current CL measurements that probe the bandgap at each position, as we do later. Here we use a relatively high beam current to create a fairly high temperature rise to more effectively smooth out the $1 \mathrm{~nm}$ variations in CL peak shift along the wire (since spectral peak shifts in this measurement are much larger than $1 \mathrm{~nm}$ ). Because we heat sink both ends of the wire, a relatively high current is needed to achieve a large red-shift. We note that in most of our nanowires a 1-2 $\mu \mathrm{m}$ region at one end shows both less CL intensity (see Figure 1c) and a slightly blue-shifted CL peak relative to the rest of the nanowire (measured at low electron beam currents), while toward the other end of the wire an abrupt increase in CL counts with a slight red-shift is observed. This is due to the doping profile introduced during nanowire growth (see Methods). We note that in the DC bridge model we neglect the interfacial thermal resistance ${ }^{61}$ between the $\mathrm{GaN}$ and the $\mathrm{Pt} / \mathrm{Cu}$ at either end, as it is small compared to the thermal resistance of $\mathrm{GaN}$, which we verify with our measurements by ensuring temperature rises are very small near the $\mathrm{Cu}$ heat sinks, as seen in Figure 3a. The interfacial thermal resistance is not always negligible between the end of the wire and the $\mathrm{Cu}$ 
substrate, which we observe in our measurements as a discontinuity between the temperature of the nanowire near the $\mathrm{Cu}$ and the $\mathrm{Cu}$ temperature, known from a thermometer in the sample stage (within $1 \mathrm{~K}$ accuracy) to which the $\mathrm{Cu}$ is thermally connected with silver paint. To overcome this, a different method can be used to measure thermal conductivity, the DC slope method. ${ }^{60}$

In the DC slope method, only one end of the wire is heat sunk (see Figure $3 \mathrm{~g}$ ) and the other end extends into the center of the hole. In this method, ${ }^{60}$ the temperature rise when the electron beam is at position $x$ away from the edge of the hole (Figure 3e) is given by

$$
\Delta T(x)=\left(R_{\mathrm{c}}+\frac{x}{A \kappa_{\mathrm{GaN}}}\right) \dot{\mathrm{Q}}
$$

where $R_{c}$ is thermal contact resistance between the wire and the $\mathrm{Cu}$ frame. If we find the slope, $s$, of this line, $\mathrm{d} \Delta T / \mathrm{d} x$, and solve for $\kappa_{\mathrm{GaN}}$, we find the expression $\kappa_{\mathrm{GaN}}=\dot{Q} /(s A)$. We determine $A$ (wire cross-sectional area) from SEM images. $\dot{Q}$ (heat flux) we determine from the measured electron beam current correcting for energy lost to backscattered or secondary electrons (determined from CASINO Monte Carlo simulations). In the case of the " $O$ " data points in Figure $3 \mathrm{~b}$, we also correct for larger electron beam sizes that resulted as a consequence of using large currents. The slope is found by fitting a line to the temperature profile of the wire sufficiently far from the Pt contacts to avoid the effect of the $\mathrm{Pt} / \mathrm{GaN}$ thermal conductivity seen in Figure 3a. We additionally subtract the doping profile (resulting from variations in intentional Si-doping during growth) of the wires found under low electron beam current ${ }^{39}$ shown in Figure $3 \mathrm{~g}$ to correct for the $\sim 1 \mathrm{~nm}$ doping variations along the wire as discussed above (see Figure S6, Methods). The profiles of three such wires are shown in Figure $3 \mathrm{~b}$ with thermal conductivities specified in the figure caption, ranging from 19 to $66 \mathrm{~W} / \mathrm{m} \cdot \mathrm{K}$ with errors ranging from $10 \%$ to $21 \%$, which derive primarily from uncertainty in $A$ due to small variations in diameter along the length of the wire and, in the case of the " $O$ " data points, from a $10 \%$ error in $\dot{Q}$ as discussed below.

The benefit of the DC slope method over the DC bridge method lies in the ability to neglect thermal contact resistances. Additionally, larger temperatures can generally be reached in wires only thermally connected on one end. Overall, DC methods suffer from strong dependence on localized doping variations. This can be overcome by extracting bandgap variations due to doping profiles with low electron beam currents, as was done in Figure $3 \mathrm{~b}$. Because both doping concentration and temperature changes cause bandgap variations, the effect of each must be determined separately. By measuring the bandgap without heating the nanowire, we can determine the bandgap variations due to doping concentrations. In these particular nanowires, the doping variations are significant. If doping is neglected, one could improperly extract a negative value for thermal conductivity from uncorrected wire $C$ thermal profile data in Supplementary Figure 6 due to a negative slope (eq 3). There is additional uncertainty that comes from the heat flux in the wire, in the case of Figure 3a and " $O$ " data points in Figure 3b. Because large currents are needed to raise temperatures for good signalto-noise ratio, larger apertures must be used in the electron column, which leads to larger spot sizes. ${ }^{63}$ This is generally negligible in comparison to the size of the electron cascade, unless a large (e.g. $1 \mathrm{~mm}$ ) aperture is used and less of the incident electron beam impinges upon the wire, adding some uncertainty to the measurements of the heat flux $\dot{Q}$. In the measurements of Figure $3 \mathrm{a}$ and $\mathrm{b}$ (" $\mathrm{O}$ ” data points only), by examining the loss of resolution in secondary electron images as a result of increased electron beam size, we calculate that only approximately $20-50 \%$ of the electron beam is reaching the nanowire without an aperture. Thus, the current actually reaching the nanowires was $64.0 \mathrm{nA}$ for Figure $3 \mathrm{a}$, and for Figure $3 \mathrm{~b}$ wires $\mathrm{A}, \mathrm{B}$, and $\mathrm{C}$ " $\mathrm{O}$ ” data points were 15.7, 11.0, and $9.3 \mathrm{nA}$, respectively. To double-check the veracity of our thermal conductivity measurements using the DC slope method, an aperture was used when collecting the " $X$ " data points in Figure $3 \mathrm{~b}$, leading to less current $(5.6,3.2$, and $3.2 \mathrm{nA}$ for wires $\mathrm{A}, \mathrm{B}$, and $\mathrm{C}$, respectively), a smaller temperature rise in the wire, but all of the measured electron beam current striking the wire in a several-nanometer-sized spot. The dependence on knowing $\dot{Q}$ to a high degree of accuracy can be overcome by using AC methods to extract thermal conductivity, as discussed in the next session.

AC Thermal Conductivity Measurements. In the AC thermal conductivity measurement technique, the column of the SEM was equipped ${ }^{64}$ with a high-frequency electrostatic beam blanker to modulate the electron current in a square wave on/off pattern. The sample configuration is the same as that used in the DC slope method described above, in which one end of the nanowire is heat sunk with SEM-deposited Pt and the other end is free (Figure $3 \mathrm{~g}$ ). In this method, we focus the electron beam on the free end of the wire for the duration of the experiment and vary the electron beam current frequency with a waveform generator between $100 \mathrm{~Hz}$ and 5 $\mathrm{MHz}$ (Figure S7). Data collection for the studied frequency range took several minutes total. Solving the $1 \mathrm{D}$ timedependent heat equation (using one Dirichlet and one timedependent-periodic Neumann boundary condition) for the quasi-steady-state temperature (after all transients have subsided) at the free end of the nanowire, temperature varies according to the expression (see Supplementary Note for more details)

$$
\begin{aligned}
T(\omega, t)= & T_{0}+\frac{4 \dot{Q} L}{A \kappa \pi} \\
& \times\left(1+\operatorname{Re}\left\{\sum_{m=1,3,5, \ldots}^{\infty} \frac{x}{L m i} \frac{\tanh \left(\sqrt{\frac{\omega L^{2} C_{p} \rho m}{2 \kappa}}(1+i)\right)}{\sqrt{\frac{\omega L^{2} C_{p} \rho m}{2 \kappa}}(1+i)} \mathrm{e}^{\mathrm{im} \omega \mathrm{t}}\right\}\right)
\end{aligned}
$$

where $T_{0}$ is the temperature of the fixed end/Cu frame, $A$ is wire cross-sectional area, $\kappa$ is the thermal conductivity (we assume uniform thermal conductivity in the wire), $\rho$ is the density of $\mathrm{GaN}^{65}\left(6150 \mathrm{~kg} / \mathrm{m}^{3}\right), C_{p}$ is the heat capacity ${ }^{65}$ (490 $\mathrm{J} / \mathrm{kg} \cdot \mathrm{K})$, and $L$ is the wire length starting from the edge of the Pt deposition. Because we use a spectrometer with a long exposure time ( $40 \mathrm{~ms}$ or longer) compared to the modulation frequency of the beam, we measure the average temperature over the half period when the electron beam is on (Figure $4 \mathrm{~b}$ ), 

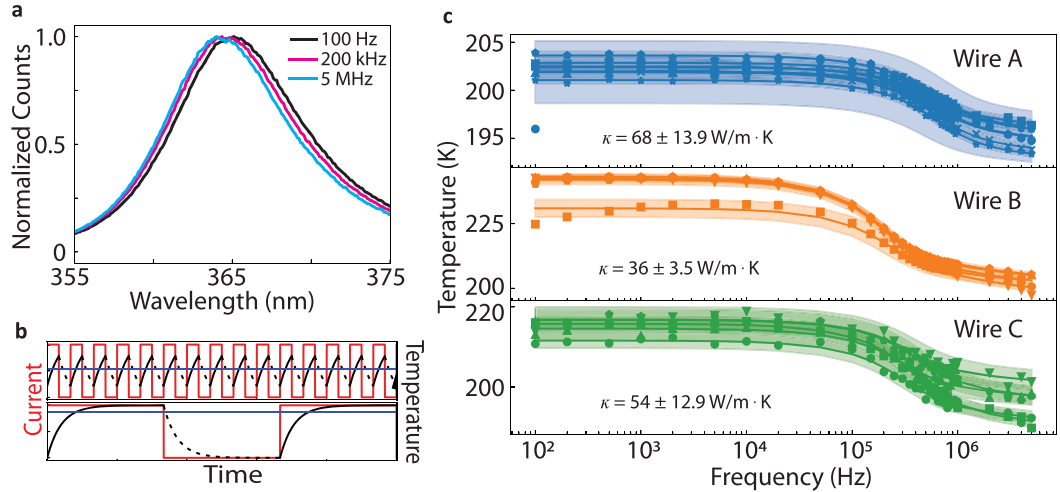

Figure 4. Cathodoluminescence thermal conductivity measurements in the frequency domain. (a) Cathodoluminescence (CL) spectra as a function of wavelength for a $100 \mathrm{~Hz}$ (black), $200 \mathrm{kHz}$ (pink), and $5 \mathrm{MHz}$ (blue) square wave electron beam excitation current. The electron beam current used in this measurement to heat/probe the nanowire was $42 \mathrm{nA}$ DC (the current measurement was taken without modulation; with modulation the DC measured current is half that value). (b) CL is only emitted when electron current (red) is flowing, so the temperature read by CL (black, solid line) will be on average (blue) higher for lower frequencies. (c) Temperature as a function of electron beam square wave frequency for the same wires from Figure $3 b$,g with one end held at a fixed temperature. Each plot shows several different data collection runs (represented by different marker types) for the same wire at slightly different locations on the end of the wire. The solid line is the best fit line to the data, and the shaded regions are 1 standard deviation of error in the fitting function. The extracted thermal conductivities are shown in each plot. Error is a combination of standard deviation of thermal conductivity extracted from plot data and percent error in measurement due to uncertainty in length measurements. The electron beam current used in this measurement to heat/ probe the nanowire was 18,29 , and $29 \mathrm{nA} \mathrm{DC}$ for wires $\mathrm{A}, \mathrm{B}$, and $\mathrm{C}$, respectively (the current measurement was taken without modulation; with modulation the DC measured current is half the given value).

$$
\begin{aligned}
\bar{T}_{\text {meas }}(\omega)= & T_{0}+\frac{4 \dot{Q} L}{A \kappa \pi} \\
& \left.\times\left(1+\frac{2}{\pi} \operatorname{Re}\left\{\sum_{m=1,3,5, \ldots}^{\infty} \frac{1}{m^{2}} \frac{\tanh \left(\sqrt{\frac{\omega L^{2} C_{p} \rho m}{2 \kappa}}(1+i)\right)}{\sqrt{\frac{\omega L^{2} C_{p} \rho m}{2 \kappa}}(1+i)}\right\}\right)\right)
\end{aligned}
$$

The sum arises from the Fourier decomposition of a square wave. We find that at low modulation frequencies (e.g., 100 $\mathrm{Hz}$ ) the time-averaged temperature of the GaN wire is higher, and therefore it has a more red-shifted CL spectrum than at higher frequencies (e.g., $5 \mathrm{MHz}$ ), as shown in Figure 4a.

Wavelength spectra for each modulation frequency were fit to extract the temperature data shown for three different wires in Figure 4c, the same wires of which were analyzed in Figure $3 \mathrm{~b}$ using the DC slope method. Several different frequency sweeps were performed for each wire at slightly different locations at the end of the wires, which correspond to the different fit curves in Figure 4c. The solid lines are fits corresponding to eq 5 . The error in thermal conductivity due to the variation in thermal conductivity extracted from the fit of each curve in Figure $4 \mathrm{c}$ is approximately $4.1 \%, 6.6 \%$, and $14 \%$ for wires A, B, and C respectively. The mean value of $L$ was taken from data from the DC slope method (i.e., where a kink in slope of temperature versus $\mathrm{x}$ appears, indicating $\mathrm{Pt}$ deposition, not shown in Figure $3 \mathrm{~d}$ but visible in Figure $3 \mathrm{a}$ ), and uncertainty was measured as the range of the visible thickening of the nanowire radius due to leakage Pt deposition, as seen in SEM images. Uncertainty in $L$ ranges from $5 \%$ to $10 \%$ for the wires. This leads to an uncertainty in thermal conductivity due only to contributions from $L$ uncertainty of $20 \%, 7.5 \%$, and $20 \%$ for wires A, B, and C, respectively. Thus, uncertainty in the $\mathrm{AC}$ method thermal conductivity measurements has the largest contribution from $L$ uncertainty. The leakage of $\mathrm{Pt}$ deposition onto the wires was the primary culprit for this uncertainty, as it is unclear where the exact location of the "fixed" temperature end of the wires is. The uncertainties can be strongly reduced by further control over the sample geometry. The DC slope method does not rely on knowledge of $L$ to extract thermal conductivity, and the DC bridge method treats the equivalent of $L$ as a fit parameter, incorporating error into the fit model.

As the incident current decreases, the average temperature will decrease across all electron beam modulation frequencies, until the current heats the wire below the error of the temperature measurement precision, giving a flat line. Supplementary Figure 8 explores this effect by focusing the electron beam off-center of the nanowire, thereby depositing less power into the nanowire. We see consistent thermal conductivities measured at different deposited powers.

The benefit of the AC method is that the value of the electron beam heat flux, $\dot{Q}$ does not need to be known in order to extract thermal conductivity, unlike in the DC methods, where these parameters are $100 \%$ correlated. As $\dot{Q}$ is determined by using a combination of measurement of the electron beam current and CASINO and Monte Carlo simulations, and can be heavily influenced by electron beam shape (in the case of large-apertured electron beams, as discussed above), uncertainties in $\dot{Q}$ are a significant source of error in the analysis. By using a spectrometer to average the frequency-dependent optical response in time, we are summing non-negligible shot noise (from electrons striking the nanowires and from generation/recombination of carriers in the $\mathrm{GaN}$ nanowires) over a wide electrical bandwidth. We could further shrink the uncertainty in thermal conductivity in these AC measurements by using a bandpass filter to isolate a small wavelength range near the bandgap CL emission and monitor amplitude modulation in this band via lock-in detection during pulsed electron beam excitation, thereby drastically improving signal-to-noise ratio as the bandwidth over which noise is summed with lock-in methods is small.

Comparison of Thermal Conductivities and Methods. The thermal conductivity of bulk GaN at room temperature 
reported in the literature is fairly high at $130-220 \mathrm{~W} / \mathrm{m} \cdot \mathrm{K} .{ }^{66,67}$ $\mathrm{GaN}$ nanowires previously studied with the suspended microchip method or with photoluminescence have reported thermal conductivity values of $13-19 \mathrm{~W} / \mathrm{m} \cdot \mathrm{K}^{29}$ for smaller diameter nanowires and $<80 \mathrm{~W} / \mathrm{m} \cdot \mathrm{K}^{58}$ for wires of similar diameter to those we studied and are therefore in agreement with our results of $19-68 \mathrm{~W} / \mathrm{m} \cdot \mathrm{K}$ found in this study. Several studies attributed the deviation from the bulk values to decreased phonon mean free path due to large mass-difference scattering from $\mathrm{Si}$ impurities. ${ }^{29,68,69}$ One study found additionally that boundary scattering, phonon confinement, and the change in nonequilibrium phonon distribution significantly contributed to the decrease in thermal conductivity in nanowires when compared to bulk. ${ }^{69} \mathrm{Si}$ impurities are present in our nanowires, as the nanowires were intentionally doped with Si during growth (see Methods). The nanoscale thermometry presented here provides avenues for detailed studies of heat flow in confined geometries.

The data shown in Figure $3 \mathrm{~d}$ are from the same wires as the data shown in Figure 4c, allowing for direct comparison between the DC slope and AC methods. The extracted thermal conductivities are within error of each other for wires $\mathrm{A}$ and $\mathrm{B}$, but not for wire $C$, although all measurements on all wires show a lower-than-bulk thermal conductivity. The thermal profile for wire $\mathrm{C}$ in Figure $3 \mathrm{~b}$ should show sections with different slope if there was a deviation in thermal conductivity along its length, but we do not see this to a significant extent. As discussed previously, the wavelength shifts due to doping must be subtracted from the wavelength shifts due to temperature to derive accurate results. If wire $\mathrm{C}$ were not completely round as it appears to be in the SEM, our estimate of the cross-sectional area could be inaccurate, leading to lower than expected thermal conductivities with the DC slope method. We did correct for drift in our frequency-dependent measurements, but it is possible that because the doping variation with position is significant at the end of this nanowire, small drifting of the beam caused a much larger effect on the bandgap shift than with more uniformly doped wires $\mathrm{A}$ and $\mathrm{B}$. Additionally, a small chip is present at the end of wire $C$, and so a small amount of drift near the edge of this chip could lead to slightly varying power deposition due to altered electron beam backscatter/transmission/absorption ratios during the measurement, altering results.

Both the DC and AC methods have their advantages and disadvantages. The DC methods allow for easier examination of the heat-sinking quality at the boundaries of the materials during data collection and have higher spatial resolution than the AC method (in our microscope). Additionally, drift during measurements is easier to spot and correct for with the DC method. The DC method is also more sensitive to variations in doping in the wire. With extra large temperature rises, as we saw in the wire used in the DC bridge method, the doping variations played less of a role in determining thermal conductivity, as temperature rises caused much larger bandgap shifts than doping variations did. In the DC slope method with smaller temperature rises, we had to correct for variations in the bandgap emission due to doping (on the order of $1 \mathrm{~nm}$ ) in the nanowire by extracting bandgap variations along the wire found with low electron beam current. On the other hand, knowledge of the thermal contact resistance was not necessary in the DC slope method, whereas it was critical in the DC bridge method. In fitting the DC data, thermal conductivity is $100 \%$ correlated with both electron beam heat flux and wire cross-sectional area, both of which had the largest sources of uncertainties.

The AC method, as it relies on the electron beam being focused on a single point on the wire, can be collected much faster. In our particular SEM electron column, spatial resolution is worse in the $\mathrm{AC}$ method due to changes in the electron beam optics necessary to place the beam into conjugate mode with the focus of the beam between the two blanking plates. ${ }^{64}$ Alternative microscope column designs with optimized beam crossover, which are available commercially, will resolve this issue. In all cases, we assume in our models that thermal conductivity (and wire cross-sectional area) is constant along the wire and not affected by doping; however, it has been shown that thermal conductivity of GaN can decrease with increased doping concentration ${ }^{68}$ (see Figure S6 for discussion). Accuracy could suffer if thermal conductivity is not uniform throughout the wire, as the mathematical model does not account for this (see Figures S6). In fitting the data, thermal conductivity can be extracted accurately without knowledge of both electron beam heat flux and wire crosssectional area in the AC method, in contrast to DC methods. The AC method may also suffer less from carrier accumulation in the bandgap, such as blue-shifting caused by the BurnsteinMoss effect. ${ }^{70,71}$ We did not see such an effect in our wires, but it could play more of a role in other materials. In all cases, we assumed heat capacity and density were constant with temperature.

The major drawback of the thermometry method presented here is the strong influence of the localized doping heterogeneity on the bandgap energy. The doping from $\mathrm{Si}$ impurities introduced intentionally during growth of the nanowires created $\sim 1 \mathrm{~nm}$ variations in the peak bandgap wavelength, which corresponds to a $\sim 50 \mathrm{~K}$ equivalent temperature variation. Doping is static (impurities cannot be added or moved), and therefore its influence on the bandgap energy can be separated from temperature changes in a straightforward manner; if the sample temperature is known (no external heating is present and a low electron beam current is used to avoid heating), any variation in peak bandgap in the sample can be attributed to doping. Maximizing accuracy of temperature calibration curves for a given sample can be done with high-resolution low-current mapping of the bandgap shifts of the entire sample at different temperatures of the heating/ cooling calibration stage. This allows for definitive separation of the doping background from the temperature shift. In this study, we have measured the calibration curve for a small region of uniform doping on a nanowire and assumed doping would shift the curve offset but not the slope, which was observed to be a reasonable assumption previously. ${ }^{42}$ Figure $3 \mathrm{~g}$ shows the bandgap variation due to doping in wires $A, B$, and $\mathrm{C}$ (the CL yield from the wire in Figures 2 and $3 \mathrm{a}$ was too low for our spectrometer to detect without a current large enough to heat the nanowire).

A modification of the AC method can be used to measure thermal conductivity without need for a calibration curve with a few assumptions. First, a low current scan must be taken to determine the peak bandgap without heating. Next, it can be assumed that the temperature scales linearly with bandgap shift, which is valid over small temperature ranges. Finally, the data can be normalized and fit with the nondimensionalized version of eq 5 given in the Supporting Information in order to extract the thermal conductivity. In short, the peak wavelength data can directly be fit to extract the thermal conductivity 
instead of first converting to temperature, eliminating the need for a calibration curve.

\section{CONCLUSIONS}

We have presented a method of cathodoluminescence nanothermometry for semiconductors along with three different methods for using this thermometry method to measure thermal conductivity of $\mathrm{GaN}$ (or other semiconductor) nanowires. CL thermometry can be used with very low currents in order to measure temperatures in situ without heating the sample, or it can be used with high currents to act additionally as a delta-function-like heat source to study thermal transport. We additionally showed that, alongside $\mathrm{GaN}$, both Si and GaAs exhibit shifts in CL bandgap emission with temperature, indicating that the temperature mapping and thermal transport measurements examined here are broadly applicable to other semiconductors and could find uses in examining integrated circuits in situ to find defects, for example. The thermal conductivity measurement methods explored here are fairly rapid and have low fabrication requirements. The existing framework for laser-based pumpprobe measurements of thermal conductivity, like time-domain thermoreflectance, could easily be translated into the SEM using CL nanothermometry, which could result in $100 \times$ better spatial resolution than these state-of-the-art methods. ${ }^{32}$ Additionally, the tunability of the penetration depth of electrons (between 10s of nanometers and microns in SEMs, controlled by the electron energy) creates possibilities for heat transport studies in nanolayers currently inaccessible in photon-based studies. Because of the high resolution, high scan speeds, and high level of control an SEM offers, CL nanothermometry-based methods offer an enticing framework in which to study phonon dynamics, ballistic transport, and near-field heat transport phenomena hitherto unmeasurable.

\section{METHODS/EXPERIMENTAL}

Nanowire Fabrication. The nanowire fabrication process using a top-down approach combining displacement Talbot lithography and selective area sublimation has been detailed previously. ${ }^{49}$ A GaN layer was grown by metalorganic-vapor-phase epitaxy on $c$-plane (0001) sapphire substrates starting with a $2 \mu \mathrm{m}$ undoped $\mathrm{GaN}$ layer followed by $5 \mu \mathrm{m}$ of Si-doped $\left(5 \times 10^{18} \mathrm{~cm}^{-3}\right)$ GaN. A $60 \mathrm{~nm}$ thick $\mathrm{Si}_{x} \mathrm{~N}_{y}$ deposited by plasma-enhanced chemical vapor deposition on top of the GaN layer was patterned with displacement Talbot lithography to get a hexagonal array of nanodisks (diameter of $515 \mathrm{~nm}$ ) with a pitch of $1.5 \mu \mathrm{m} .{ }^{72}$ The sample then underwent selective area sublimation between 900 and $920{ }^{\circ} \mathrm{C}$ in a molecular beam epitaxy chamber for $8 \mathrm{~h}$ in order to define the $7 \mu \mathrm{m}$ nanowires. The $\mathrm{Si}_{x} \mathrm{~N}_{y}$ mask was etched using a HF-based solution. Some wires shown in this article are slightly shorter than $7 \mu \mathrm{m}$ due to breaking when separated from the growth substrate or variations in the sublimation rate due to the temperature gradient between the center and the edge of the wafer.

\section{ASSOCIATED CONTENT}

\section{SI Supporting Information}

The Supporting Information is available free of charge at https://pubs.acs.org/doi/10.1021/acsnano.1c00850.

Nanowire damage analysis; Monte Carlo simulations; peak wavelength versus temperature calibration curves; doping effects on peak wavelength; frequency roll-off of beam blanker; thermal modal calculations; varied electron beam current temperature response with frequency (PDF)

\section{AUTHOR INFORMATION}

\section{Corresponding Authors}

Kelly W. Mauser - Center for Nanophotonics, NWO-Institute AMOLF, Amsterdam 1098, XG, The Netherlands; 이이.org/0000-0001-9903-8559; Email: k.mauser@ amolf.nl

Albert Polman - Center for Nanophotonics, NWO-Institute AMOLF, Amsterdam 1098, XG, The Netherlands; 다. orcid.org/0000-0002-0685-3886; Email: a.polman@ amolf.nl

\section{Authors}

Magdalena Solà-Garcia - Center for Nanophotonics, NWOInstitute AMOLF, Amsterdam 1098, XG, The Netherlands; (1) orcid.org/0000-0002-2614-1050

Matthias Liebtrau - Center for Nanophotonics, NWOInstitute AMOLF, Amsterdam 1098, XG, The Netherlands; - orcid.org/0000-0002-2374-696X

Benjamin Damilano - Université Côte d'Azur, CNRS, CRHEA, Nice 06103, France

Pierre-Marie Coulon - University of Bath, Bath BA2 7AY, United Kingdom

Stéphane Vézian - Université Côte d'Azur, CNRS, CRHEA, Nice 06103, France

Philip A. Shields - University of Bath, Bath BA2 7AY, United Kingdom; (1) orcid.org/0000-0003-0517-132X

Sophie Meuret - CEMES/CNRS, Toulouse 31055, France; (1) orcid.org/0000-0001-8511-9972

Complete contact information is available at:

https://pubs.acs.org/10.1021/acsnano.1c00850

\section{Author Contributions}

K.M., A.P., and S.M. conceived the experiment, B.D., P.C., S.V., and P.S. fabricated the nanowires, K.M. measured the data, K.M. analyzed the data, S.M., M.S.G., M.L., and K.M. built the measurement systems, and all authors contributed to the manuscript.

\section{Notes}

The authors declare the following competing financial interest(s): A.P. is co-founder and co-owner of Delmic BV, a company that produces the cathodoluminescence system that was used in this work.

This paper is based on a previously available preprint: Mauser, K. W., Solà-Garcia, M., Liebtrau, M., Damilano, B., Coulon, P. M., Vézian, S., Shields, P., Meuret, S., Polman, A., Probing Nanoscale Thermal Transport with Cathodoluminescence Thermometry. 2020. arXiv:2012.14890. arXiv. https://arxiv. org/abs/2012.14890 (accessed June 10, 2021).

\section{ACKNOWLEDGMENTS}

The authors would like to thank V. Neder and I. Hoogsteder for assistance with electron-assisted Pt deposition. This work is part of the research program of AMOLF, which is partly financed by the Dutch Research Council (NWO). This project has received funding from the European Research Council (ERC) under the European Union's Horizon 2020 research and innovation program (grant agreement no. 695343). The project is also supported by the European Union's Horizon 2020 research and innovation program under grant agreement no. 101017720 (FET-Proactive EBEAM). This work has been supported by the French National Research Agency (ANR) through the project NAPOLI (ANR-18-CE24-0022) and 
through the project ECHOMELO (ANR-19-CE30-0008 ECHOMELO). The work is also supported by UK Research \& Innovation: EPSRC grant no. EP/M015181/1.

\section{REFERENCES}

(1) Dauler, E. A.; Grein, M. E.; Kerman, A. J.; Marsili, F.; Miki, S.; Nam, S. W.; Shaw, M. D.; Terai, H.; Verma, V. B.; Yamashita, T. Review of Superconducting Nanowire Single-Photon Detector System Design Options and Demonstrated Performance. Opt. Eng. 2014, 53 (8), 081907.

(2) Zimmler, M. A.; Capasso, F.; Müller, S.; Ronning, C. Optically Pumped Nanowire Lasers: Invited Review. Semicond. Sci. Technol. 2010, 25 (2), 024001.

(3) Hamid Elsheikh, M.; Shnawah, D. A.; Sabri, M. F. M.; Said, S. B. M.; Haji Hassan, M.; Ali Bashir, M. B.; Mohamad, M. A Review on Thermoelectric Renewable Energy: Principle Parameters That Affect Their Performance. Renewable Sustainable Energy Rev. 2014, 30, 337355.

(4) Pop, E.; Sinha, S.; Goodson, K. E. Heat Generation and Transport in Nanometer-Scale Transistors. Proc. IEEE 2006, 94 (8), $1587-1601$

(5) Yang, F.; Dames, C. Mean Free Path Spectra as a Tool to Understand Thermal Conductivity in Bulk and Nanostructures. Phys. Rev. B: Condens. Matter Mater. Phys. 2013, 87 (3), 035437.

(6) Poudel, B.; Hao, Q.; Ma, Y.; Lan, Y.; Minnich, A.; Yu, B.; Yan, X.; Wang, D.; Muto, A.; Vashaee, D.; Chen, X.; Liu, J.; Dresselhaus, M. S.; Chen, G.; Ren, Z. High-Thermoelectric Performance of Nanostructured Bismuth Antimony Telluride Bulk Alloys. Science 2008, 320 (5876), 634-638.

(7) Luckyanova, M. N.; Garg, J.; Esfarjani, K.; Jandl, A.; Bulsara, M. T.; Schmidt, A. J.; Minnich, A. J.; Chen, S.; Dresselhaus, M. S.; Ren, Z.; Fitzgerald, E. A.; Chen, G. Coherent Phonon Heat Conduction in Superlattices. Science 2012, 338 (6109), 936-939.

(8) Yang, B.; Chen, G. Partially Coherent Phonon Heat Conduction in Superlattices. Phys. Rev. B: Condens. Matter Mater. Phys. 2003, 67 (19), 195311.

(9) Minnich, A. J.; Chen, G.; Mansoor, S.; Yilbas, B. S. Quasiballistic Heat Transfer Studied Using the Frequency-Dependent Boltzmann Transport Equation. Phys. Rev. B: Condens. Matter Mater. Phys. 2011, 84 (23), 235207.

(10) Huang, B.-W.; Hsiao, T.-K.; Lin, K.-H.; Chiou, D.-W.; Chang, C.-W. Length-Dependent Thermal Transport and Ballistic Thermal Conduction. AIP Adv. 2015, 5 (5), 053202.

(11) Dresselhaus, M. S.; Chen, G.; Tang, M. Y.; Yang, R. G.; Lee, H.; Wang, D. Z.; Ren, Z. F.; Fleurial, J.-P.; Gogna, P. New Directions for Low-Dimensional Thermoelectric Materials. Adv. Mater. 2007, 19 (8), 1043-1053.

(12) Sood, A.; Xiong, F.; Chen, S.; Cheaito, R.; Lian, F.; Asheghi, M.; Cui, Y.; Donadio, D.; Goodson, K. E.; Pop, E. Quasi-Ballistic Thermal Transport across $\mathrm{MoS}_{2}$ Thin Films. Nano Lett. 2019, 19 (4), 2434-2442.

(13) Hu, Y.; Zeng, L.; Minnich, A. J.; Dresselhaus, M. S.; Chen, G. Spectral Mapping of Thermal Conductivity through Nanoscale Ballistic Transport. Nat. Nanotechnol. 2015, 10 (8), 701-706.

(14) Brites, C. D. S.; Lima, P. P.; Silva, N. J. O.; Millán, A.; Amaral, V. S.; Palacio, F.; Carlos, L. D. Thermometry at the Nanoscale. Nanoscale 2012, 4 (16), 4799-4829.

(15) Luo, T.; Chen, G. Nanoscale Heat Transfer - from Computation to Experiment. Phys. Chem. Chem. Phys. 2013, 15 (10), 3389-3412.

(16) Yue, Y.; Wang, X. Nanoscale Thermal Probing. Nano Rev. 2012, 3, 1-11.

(17) Christofferson, J.; Maize, K.; Ezzahri, Y.; Shabani, J.; Wang, X.; Shakouri, A. Microscale and Nanoscale Thermal Characterization Techniques. J. Electron. Packag. 2008, 130 (4), 3-9.

(18) Yue, Y.; Chen, X.; Wang, X. Noncontact Sub-10 Nm Temperature Measurement in Near-Field Laser Heating. ACS Nano 2011, 5 (6), 4466-4475.
(19) Vertikov, A.; Kuball, M.; Nurmikko, A. V.; Maris, H. J. TimeResolved Pump-Probe Experiments with Subwavelength Lateral Resolution. Appl. Phys. Lett. 1996, 69 (17), 2465-2467.

(20) Varesi, J.; Majumdar, A. Scanning Joule Expansion Microscopy at Nanometer Scales. Appl. Phys. Lett. 1998, 72 (1), 37-39.

(21) Shi, L.; Plyasunov, S.; Bachtold, A.; McEuen, P. L.; Majumdar, A. Scanning Thermal Microscopy of Carbon Nanotubes Using BatchFabricated Probes. Appl. Phys. Lett. 2000, 77 (26), 4295-4297.

(22) Idrobo, J. C.; Lupini, A. R.; Feng, T.; Unocic, R. R.; Walden, F. S.; Gardiner, D. S.; Lovejoy, T. C.; Dellby, N.; Pantelides, S. T.; Krivanek, O. L. Temperature Measurement by a Nanoscale Electron Probe Using Energy Gain and Loss Spectroscopy. Phys. Rev. Lett. 2018, 120 (9), 095901.

(23) Yan, X.; Liu, C.; Gadre, C. A.; Dai, S.; Gu, L.; Yu, K.; Aoki, T.; $\mathrm{Wu}, \mathrm{R}$; Pan, X. Unexpected Strong Thermally Induced Phonon Energy Shift for Mapping Local Temperature. Nano Lett. 2019, 19 (10), 7494-7502.

(24) Mecklenburg, M.; Hubbard, W. A.; White, E. R.; Dhall, R.; Cronin, S. B.; Aloni, S.; Regan, B. C. Nanoscale Temperature Mapping in Operating Microelectronic Devices. Science 2015, 347 (6222), 629-632.

(25) Cahill, D. G. Thermal Conductivity Measurement from 30 to 750 K: The $3 \omega$ Method. Rev. Sci. Instrum. 1990, 61 (2), 802-808.

(26) Birge, N. O. Specific-Heat Spectroscopy of Glycerol and Propylene Glycol near the Glass Transition. Phys. Rev. B: Condens. Matter Mater. Phys. 1986, 34 (3), 1631-1642.

(27) Kim, P.; Shi, L.; Majumdar, A.; McEuen, P. L. Thermal Transport Measurements of Individual Multiwalled Nanotubes. Phys. Rev. Lett. 2001, 87 (21), 215502.

(28) Shi, L.; Li, D.; Yu, C.; Jang, W.; Kim, D.; Yao, Z.; Kim, P.; Majumdar, A. Measuring Thermal and Thermoelectric Properties of One-Dimensional Nanostructures Using a Microfabricated Device. J. Heat Transfer 2003, 125 (5), 881-888.

(29) Guthy, C.; Nam, C.-Y.; Fischer, J. E. Unusually Low Thermal Conductivity of Gallium Nitride Nanowires. J. Appl. Phys. 2008, 103 (6), 064319.

(30) Cahill, D. G. Analysis of Heat Flow in Layered Structures for Time-Domain Thermoreflectance. Rev. Sci. Instrum. 2004, 75 (12), $5119-5122$

(31) Wilson, R. B.; Cahill, D. G. Anisotropic Failure of Fourier Theory in Time-Domain Thermoreflectance Experiments. Nat. Commun. 2014, 5 (1), 5075.

(32) Bahk, J.; Shakouri, A. Ultra-Fast Thermoreflectance Imaging for Electronic, Optoelectronic, and Thermal Devices. In 2019 IEEE BiCMOS and Compound semiconductor Integrated Circuits and Technology Symposium (BCICTS) Proceedings of the 2019 IEEE BiCMOS and Compound semiconductor Integrated Circuits and Technology Symposium (BCICTS); IEEE, November 3-6, 2019; Nashville, TN, USA, 2019; pp 1-7.

(33) Jiang, P.; Qian, X.; Yang, R. Tutorial: Time-Domain Thermoreflectance (TDTR) for Thermal Property Characterization of Bulk and Thin Film Materials. J. Appl. Phys. 2018, 124 (16), 161103.

(34) Klein, C. A. Radiation Ionization Energies in Semiconductors: Speculations about the Role of Plasmons. J. Phys. Soc. Jpn. Suppl. 1966, 21, 307-311.

(35) Rothwarf, A. Plasmon Theory of Electron-hole Pair Production: Efficiency of Cathode Ray Phosphors. J. Appl. Phys. 1973, 44 (2), $752-756$.

(36) Egerton, R. F. Electron Energy-Loss Spectroscopy in the TEM. Rep. Prog. Phys. 2009, 72 (1), 016502.

(37) Remond, G. Applications of Cathodoluminescence in Mineralogy. J. Lumin. 1977, 15 (2), 121-155.

(38) Christen, J.; Grundmann, M.; Bimberg, D. Scanning Cathodoluminescence Microscopy: A Unique Approach to AtomicScale Characterization of Heterointerfaces and Imaging of Semiconductor Inhomogeneities. J. Vac. Sci. Technol., B: Microelectron. Process. Phenom. 1991, 9 (4), 2358-2368. 
(39) Chen, H.-L.; Himwas, C.; Scaccabarozzi, A.; Rale, P.; Oehler, F.; Lemaître, A.; Lombez, L.; Guillemoles, J.-F.; Tchernycheva, M.; Harmand, J.-C.; Cattoni, A.; Collin, S. Determination of N-Type Doping Level in Single GaAs Nanowires by Cathodoluminescence. Nano Lett. 2017, 17 (11), 6667-6675.

(40) García de Abajo, F. J. Optical Excitations in Electron Microscopy. Rev. Mod. Phys. 2010, 82 (1), 209-275.

(41) Coenen, T.; Brenny, B. J. M.; Vesseur, E. J.; Polman, A. Cathodoluminescence Microscopy: Optical Imaging and Spectroscopy with Deep-Subwavelength Resolution. MRS Bull. 2015, 40 (4), 359-365.

(42) Davidson, S. M.; Vaidya, A. W. High Resolution Temperature Measurements in Microwave Devices. In Proceedings of the International Symposium on Gallium Arsenide and Related Compounds; 33a; IoP: Edinburgh, 1977; pp 287-295.

(43) Caldwell, M. A.; Haynor, B.; Aloni, S.; Ogletree, D. F.; Wong, H.-S. P.; Urban, J. J.; Milliron, D. J. Spectroscopic Evidence for Exceptional Thermal Contribution to Electron Beam-Induced Fragmentation. J. Phys. Chem. C 2010, 114 (50), 22064-22068.

(44) Yacobi, B. G.; Holt, D. B. Cathodoluminescence Scanning Electron Microscopy of Semiconductors. J. Appl. Phys. 1998, 59 (4), R1.

(45) Zhao, S.; Nguyen, H. P. T.; Kibria, Md. G.; Mi, Z. III-Nitride Nanowire Optoelectronics. Prog. Quantum Electron. 2015, 44, 14-68.

(46) Hetzl, M.; Wierzbowski, J.; Hoffmann, T.; Kraut, M.; Zuerbig, V.; Nebel, C. E.; Müller, K.; Finley, J. J.; Stutzmann, M. GaN Nanowire Arrays for Efficient Optical Read-Out and Optoelectronic Control of NV Centers in Diamond. Nano Lett. 2018, 18 (6), 36513660.

(47) Huang, Y.; Duan, X.; Cui, Y.; Lieber, C. M. Gallium Nitride Nanowire Nanodevices. Nano Lett. 2002, 2 (2), 101-104.

(48) Johnson, J. C.; Choi, H.-J.; Knutsen, K. P.; Schaller, R. D.; Yang, P.; Saykally, R. J. Single Gallium Nitride Nanowire Lasers. Nat. Mater. 2002, 1 (2), 106-110.

(49) Damilano, B.; Coulon, P.-M.; Vézian, S.; Brändli, V.; Duboz, J.Y.; Massies, J.; Shields, P. A. Top-Down Fabrication of GaN NanoLaser Arrays by Displacement Talbot Lithography and Selective Area Sublimation. Appl. Phys. Express 2019, 12 (4), 045007.

(50) Brenny, B. J. M. Probing Light Emission at the Nanoscale with Cathodoluminescence; Ph.D. thesis, University of Amsterdam, Amsterdam, NL, 2016.

(51) Demers, H.; Poirier-Demers, N.; Couture, A. R.; Joly, D.; Guilmain, M.; de Jonge, N.; Drouin, D. Three-Dimensional Electron Microscopy Simulation with the CASINO Monte Carlo Software. Scanning 2011, 33 (3), 135-146.

(52) Yuan, P.; Wu, J. Y.; Ogletree, D. F.; Urban, J. J.; Dames, C.; Ma, Y. Adapting the Electron Beam from SEM as a Quantitative Heating Source for Nanoscale Thermal Metrology. Nano Lett. 2020, 20 (5), 3019-3029.

(53) Aiyiti, A.; Bai, X.; Wu, J.; Xu, X.; Li, B. Measuring the Thermal Conductivity and Interfacial Thermal Resistance of Suspended $\mathrm{MoS}_{2}$ Using Electron Beam Self-Heating Technique. Science Bulletin 2018, 63 (7), 452-458.

(54) Liu, D.; Xie, R.; Yang, N.; Li, B.; Thong, J. T. L. Profiling Nanowire Thermal Resistance with a Spatial Resolution of Nanometers. Nano Lett. 2014, 14 (2), 806-812.

(55) Wang, Z.; Xie, R.; Bui, C. T.; Liu, D.; Ni, X.; Li, B.; Thong, J. T. L. Thermal Transport in Suspended and Supported Few-Layer Graphene. Nano Lett. 2011, 11 (1), 113-118.

(56) O'Donnell, K. P.; Chen, X. Temperature Dependence of Semiconductor Band Gaps. Appl. Phys. Lett. 1991, 58 (25), 29242926.

(57) Varshni, Y. P. Temperature Dependence of the Energy Gap in Semiconductors. Physica 1967, 34 (1), 149-154.

(58) Westover, T.; Jones, R.; Huang, J. Y.; Wang, G.; Lai, E.; Talin, A. A. Photoluminescence, Thermal Transport, and Breakdown in Joule-Heated GaN Nanowires. Nano Lett. 2009, 9 (1), 257-263.

(59) Shan, W.; Schmidt, T. J.; Yang, X. H.; Hwang, S. J.; Song, J. J.; Goldenberg, B. Temperature Dependence of Interband Transitions in
GaN Grown by Metalorganic Chemical Vapor Deposition. Appl. Phys. Lett. 1995, 66 (8), 985-987.

(60) Doerk, G. S.; Carraro, C.; Maboudian, R. Single Nanowire Thermal Conductivity Measurements by Raman Thermography. ACS Nano 2010, 4 (8), 4908-4914.

(61) Hsu, I.-K.; Kumar, R.; Bushmaker, A.; Cronin, S. B.; Pettes, M. T.; Shi, L.; Brintlinger, T.; Fuhrer, M. S.; Cumings, J. Optical Measurement of Thermal Transport in Suspended Carbon Nanotubes. Appl. Phys. Lett. 2008, 92 (6), 063119.

(62) COMSOL Multiphysics, v5.2; COMSOL AB: Stockholm, Sweden, 2015.

(63) Reimer, L. Scanning Electron Microscopy: Physics of Image Formation and Microanalysis, 2nd ed.; Springer Series in Optical Sciences; Springer-Verlag: Berlin Heidelberg, 1998.

(64) Meuret, S.; SolàGarcia, M.; Coenen, T.; Kieft, E.; Zeijlemaker, H.; Lätzel, M.; Christiansen, S.; Woo, S. Y.; Ra, Y. H.; Mi, Z.; Polman, A. Complementary Cathodoluminescence Lifetime Imaging Configurations in a Scanning Electron Microscope. Ultramicroscopy 2019, $197,28-38$

(65) Bougrov, V.; Levinshtein, M.; Rumyantsev, S.; Zubrilov, A. In Properties of Advanced Semiconductor Materials: GaN, AIN, InN, BN, $\mathrm{SiC}, \mathrm{SiGe}$; Levinshtein, M., Rumyantsev, S., Shur, M., Eds.; John Wiley \& Sons, Inc.: New York, 2001.

(66) Sichel, E. K.; Pankove, J. I. Thermal Conductivity of GaN, 25360 K. J. Phys. Chem. Solids 1977, 38, 330.

(67) Jeżowski, A.; Danilchenko, B. A.; Boćkowski, M.; Grzegory, I.; Krukowski, S.; Suski, T.; Paszkiewicz, T. Thermal Conductivity of GaN Crystals in 4.2-300 K Range. Solid State Commun. 2003, 128 (2), 69-73.

(68) Florescu, D. I.; Asnin, V. M.; Pollak, F. H.; Molnar, R. J.; Wood, C. E. C. High Spatial Resolution Thermal Conductivity and Raman Spectroscopy Investigation of Hydride Vapor Phase Epitaxy Grown N-GaN/Sapphire (0001): Doping Dependence. J. Appl. Phys. 2000, 88 (6), 3295-3300.

(69) Zou, J. Lattice Thermal Conductivity of Freestanding Gallium Nitride Nanowires. J. Appl. Phys. 2010, 108 (3), 034324.

(70) Burstein, E. Anomalous Optical Absorption Limit in InSb. Phys. Rev. 1954, 93 (3), 632-633.

(71) Moss, T. S. The Interpretation of the Properties of Indium Antimonide. Proc. Phys. Soc., London, Sect. B 1954, 67 (10), 775.

(72) Coulon, P.-M.; Damilano, B.; Alloing, B.; Chausse, P.; Walde, S.; Enslin, J.; Armstrong, R.; Vézian, S.; Hagedorn, S.; Wernicke, T.; Massies, J.; Zúñiga-Pérez, J.; Weyers, M.; Kneissl, M.; Shields, P. A. Displacement Talbot Lithography for Nano-Engineering of III-Nitride Materials. Microsystems \& Nanoengineering 2019, 5 (1), 1-12. 